\title{
Global Galactic distribution of the 1.275 MeV $\gamma$-ray line emission
}

\author{
A. F. Iyudin ${ }^{1}$, K. Bennett ${ }^{2}$, G. G. Lichti ${ }^{1}$, J. Ryan ${ }^{3}$, and V. Schönfelder ${ }^{1}$ \\ 1 Max-Planck-Institut für extraterrestrische Physik, Postfach 1312, 85741 Garching, Germany \\ e-mail: ani@mpe.mpg.de \\ 2 Research and Scientific Support Division, ESTEC, 2200 AG Noordwijk, The Netherlands \\ ${ }^{3}$ University of New Hampshire, Institute for Studies of Earth, Oceans and Space, Durham NH 03284, USA
}

Received 5 July 2005 / Accepted 22 July 2005

\section{ABSTRACT}

We present results of the analysis of $1.275 \mathrm{MeV} \gamma$-ray line global distribution derived from the all-sky data accumulated by COMPTEL on board the Compton Gamma Ray Observatory (CGRO) from 1991 to 1997. Previously the $1.275 \mathrm{MeV} \gamma$-ray line was believed to be largely produced in the decay of radioactive isotope ${ }^{22} \mathrm{Na}$ that is synthesised in the classical nova (CN) thermonuclear runaway (TNR). Another way to produce the $1.275 \mathrm{MeV}$ line emission is via the excitation of ${ }^{22} \mathrm{Ne}$-nuclei, e.g. through the low-energy cosmic ray interactions with the nuclei of the interstellar matter that lead to the production of ${ }^{22} \mathrm{Ne}^{*}$, or of ${ }^{22} \mathrm{Na}$. This scenario, as we now believe, can be dominant in contributing to the total $1.275 \mathrm{MeV} \gamma$-ray line emission from the galactic bulge.

Unfortunately, systematic uncertainties in the analysis of the COMPTEL data hinder a clear distinction between two alternative scenarios.

Key words. gamma-rays: observations - Galaxy: abundances - Galaxy: bulge - ISM: abundances - ISM: cosmic rays -

nuclear reactions, nucleosynthesis, abundances

\section{Introduction}

Penetrating 1.275 MeV $\gamma$-ray photons provide a means to study individual novae and the distribution of novae in the Galaxy. The $1.275 \mathrm{MeV} \gamma$-ray line is associated with the decay of radioactive isotope ${ }^{22} \mathrm{Na}$ that is synthesised in the classical nova (CN) thermonuclear runaway (Clayton \& Hoyle 1974). Potentially, this approach allows us to derive the rather uncertain galactic novae rate by analysing the integrated $1.275 \mathrm{MeV}$ line emission from the disk and/or bulge population. The accumulation of ${ }^{22} \mathrm{Na}$ ejected by frequent novae may manifest itself as an extended 1.275 MeV $\gamma$-ray line emission from the bulge.

Alternatively, $1.275 \mathrm{MeV}$ line emission can be produced by the low-energy cosmic ray interactions with the abundant elements of the interstellar matter (ISM) in the galactic bulge with end-products such as ${ }^{22} \mathrm{Ne}^{*}$, and ${ }^{22} \mathrm{Na}$. In this case the 1.275 MeV line can also be observed as an extended emission region.

The classical nova outburst is modelled as a thermonuclear runaway (TNR) in the accreted hydrogen-rich envelope of the white dwarf companion of a close binary system (e.g. Truran 1982). In general, observations of novae support such models (Gallagher \& Starrfield 1978; Gehrz et al. 1998).

According to the TNR model, novae are expected to produce radioactive isotopes of ${ }^{7} \mathrm{Be},{ }^{22} \mathrm{Na}$ and ${ }^{26} \mathrm{Al}$ (Truran 1982; Gehrz et al. 1998). These isotopes decay to ${ }^{7} \mathrm{Li},{ }^{22} \mathrm{Ne}$ and ${ }^{26} \mathrm{Mg}$, emitting $\gamma$-rays at energies $0.478 \mathrm{MeV}, 1.275 \mathrm{MeV}$ and $1.809 \mathrm{MeV}$, respectively. None of these lines has been detected from the individual classical novae, either by SMM (Leising et al. 1988), or by COMPTEL and OSSE instruments on-board the Compton Gamma-Ray Observatory (Iyudin et al. 1995; Leising 1997; Harris et al. 2000; Iyudin et al. 2000). These results are consistent with the latest predictions of the nova models (Gomez-Gomar et al. 1998; Jose \& Hernanz 1998; Hernanz et al. 1999; Jose et al. 1999; Starrfield et al. 2000), although the large uncertainty in the predicted ${ }^{22} \mathrm{Na}$ yield remains (Jose et al. 1999; Starrfield et al. 2000; Iliadis et al. 2002; Bishop et al. 2003; D'Auria et al. 2004; Jenkins et al. 2004).

In the following we present the first all-sky map in the 1.275 MeV $\gamma$-ray line emission derived from the data accumulated by COMPTEL on board the Compton Gamma Ray Observatory (CGRO) from 1991 to 1997. The map contains an extended emission region, which concentrates towards the galactic bulge. The extended emission in the $1.275 \mathrm{MeV}$ line can as well be explained in the context of cosmic ray interactions with the abundant constituents of the ISM in the galactic bulge. This interpretation is preferable because the $1.275 \mathrm{MeV}$ line has not been detected from any of the relatively bright and nearby CNe (Iyudin et al. 1995; Leising 1997; Iyudin et al. 2000).

We analyse $1.275 \mathrm{MeV}$ all-sky map and bulge spectra in order to address possible implications for the scenario of the line emission production by low-energy cosmic rays interacting with the bulge ISM, taking into account the known systematic uncertainties in the analysis of the COMPTEL data. 

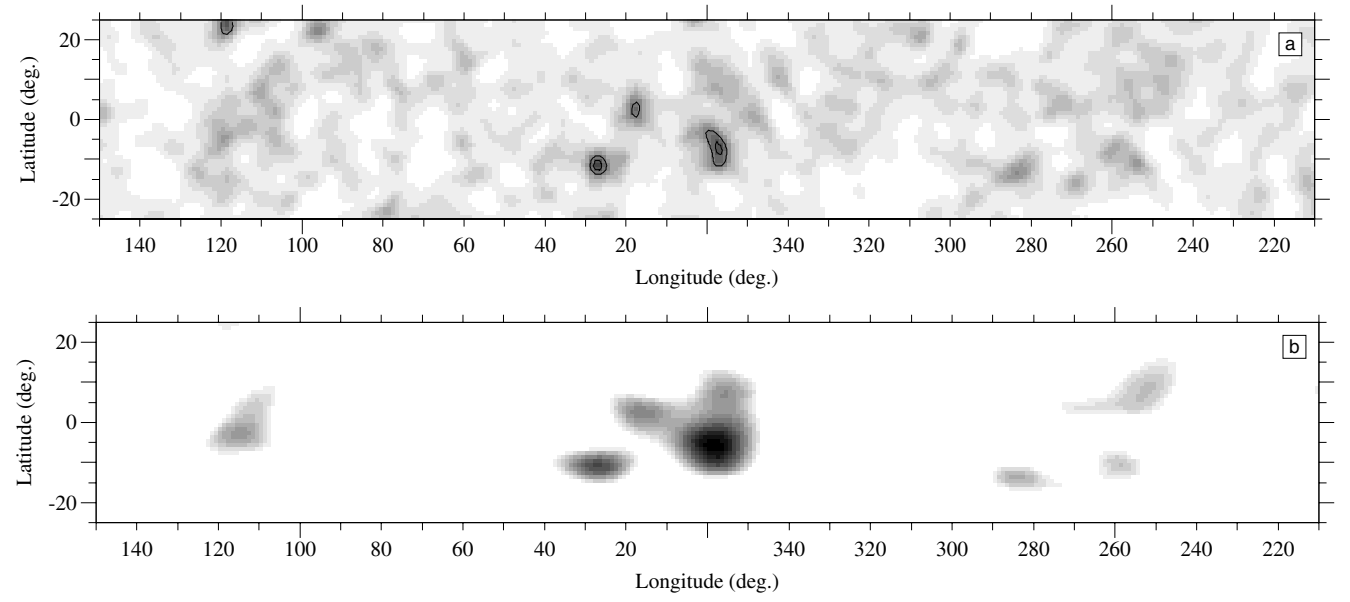

Fig. 1. a) The maximum entropy (ME) flux map of the galactic plane in the light of the $1.275 \mathrm{MeV}$ line emission (continuum emission is suppressed); b) the ME-map of the galactic centre region in the $1.275 \mathrm{MeV}$ line emission smoothed with a 2-D Gaussian (continuum emission is suppressed). The likelihood values of excesses in the galactic centre region can be read from the map shown in Fig. 2. All COMPTEL observations up to April 1997 were used for these maps.

\section{Observations and data analysis}

The combination of imaging and spectroscopic capabilities of COMPTEL (Schönfelder et al. 1993), provided a unique opportunity to measure line emission from point-like sources or from extended regions (e.g. the Galactic bulge).

The large field of view of COMPTEL facilitated serendipitous detections of galactic novae (Iyudin et al. 1995, 1999, 2001). No positive detection can be reported for any of the observed neon-type novae. An average $2 \sigma$ upper limit on the ejected ${ }^{22} \mathrm{Na}$ mass for any neon-type nova in the Galactic disk of $3.7 \times 10^{-8} M_{\odot}$ was derived (Iyudin et al. 1995), which is lower than those previously derived by HEAO-3 (Mahoney et al. 1982), SMM (Leising et al. 1988) or OSSE (Leising et al. 1993).

All data accumulated by COMPTEL up to the second CGRO reboost (24.03.1997) were used for the production of a first all-sky map in the $1.275 \mathrm{MeV}$ line emission. We restricted the analysis of this map to galactic latitudes of $\leq 30^{\circ}$.

The imaging analysis and flux evaluation were performed in a $\pm 2 \sigma$ energy window around the $1.275 \mathrm{MeV}$ line, where $\sigma$ is the instrumental energy resolution for this line. For the detection of weak sources it is essential to optimise the signal-tonoise $(\mathrm{S} / \mathrm{N})$ ratio of COMPTEL, which is at the percent level. A powerful tool to optimise the $\mathrm{S} / \mathrm{N}$ ratio is event selection. The most critical selection parameters are the time-of-flight (ToF) window of accepted events, the upper bound of the scatter angle $(\bar{\varphi})$ values, and the minimum Earth-horizon angle $\zeta$, which helps to suppress the contribution of the atmospheric Earthhorizon events to the total background. Selections were made on the ToF window of $115-130, \zeta \geq 10^{\circ}$, and the omission of the "faulty" D2-modules, i.e. three out of twelve operating D2-modules that have one out of seven photomultipliers (PMs) switched off.

Another important requirement for the sound detection of a source is the correct handling of the background underlying the source signal. Different background models were developed within the COMPTEL team for this purpose. One approach is to model the background in the line interval from the data at adjacent energy intervals. A second method derives the background model from the line energy interval itself. This is accomplished by applying a low-pass filter to the data in the line interval, which smoothes the photons' distribution and suppresses (in first approximation) the source signature (e.g. Bloemen et al. 1994). By applying iterations of this process the background estimate can be improved further (Bloemen et al. 1999). Both methods have been extensively used during the study of the ${ }^{22} \mathrm{Na}$ line emission from novae. Note, that for any of the background models used, a systematic uncertainty in the line flux (or flux upper limit) value remains up to $\sim 20 \%$ of the flux at the instrument sensitivity limit. This systematic effect is produced by the underlying continuum emission and small differences of the event distribution in the instrument coordinate space.

For imaging analysis we used two different methods. In the analysis of the global galactic flux distribution in the 1.275 MeV line emission a maximum entropy (ME) method was applied (Figs. 1a,b). To derive fluxes or upper limits from a point-like excess the maximum-likelihood (ML) method was extensively used (Fig. 2).

In this study, the spectral analysis was performed with gamma-quanta selected with a scatter direction and scatter angle compatible with circular regions of $2^{\circ}$ radii centred at the position of the excesses detected in the ME- or ML-maps. This approach was described by Iyudin et al. (1994), and was used through the subsequent studies of $\gamma$-ray lines from different point sources (Iyudin et al. 1995, 1998, 2000).

Background spectra were generated using two different methods. First, by producing spectra for different observations of the North Galactic Pole (NGP) region applying the same event selections as for the source region and avoiding regions possibly contaminated by emission from Loop I and Loop IV 


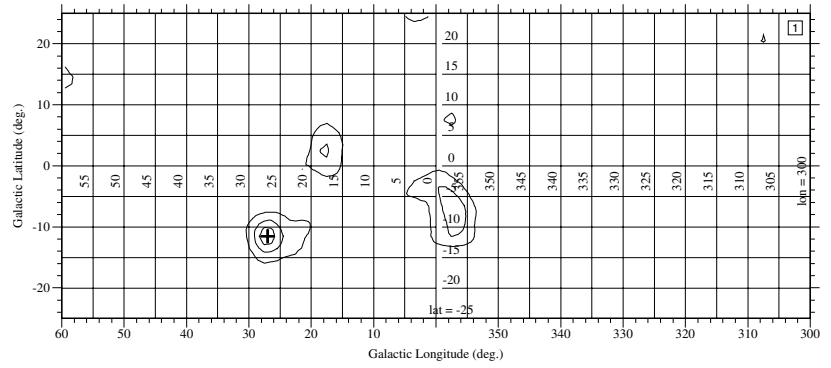

Fig. 2. The maximum-likelihood map of the galactic centre region in the $1.275 \mathrm{MeV}$ line emission. COMPTEL observations up to April 1997 were used. The position of the Aquila excess is marked by the cross. Contour lines show likelihood ratio values of 9,16 , and, additionally of a 21.6 (1 sigma error box) for the Aquila excess.

supernova remnants. Afterwards spectra of high-latitude observations of the South Galactic Pole (SGP) were co-added. This background model is preferable for the spectral analysis of the extended regions if compared with a standard background model (Iyudin et al. 1994).

The standard background model, which was extensively used in the spectral analysis of point-like line-emission excesses (Iyudin et al. 1994, 1995, 1998), is constructed using gamma-quanta collected from the same direction with large $\left(10^{\circ}\right)$ radii centred on the line excess position. Unfortunately, such a background model is not useful when applied to the extended emission regions such as the galactic bulge. Therefore, we have performed the spectral analysis of the bulge using the NGP+SGP background model, normalised to the total number of counts in the source spectrum. and with an energy binning of $20 \mathrm{keV}$.

\section{Results and discussion}

To derive the integrated flux in the $1.275 \mathrm{MeV}$ line from the galactic bulge we have analysed the all-sky maximum entropy map in $1.275 \mathrm{MeV}$ line emission (Fig. 1a). Below we used this map to produce latitude and longitude profiles of the emission in the $1.275 \mathrm{MeV}$ line. Figure $1 \mathrm{~b}$ shows a version of the ME map smoothed with a 2D Gaussian filter. We have used a filter with $\sigma_{b}=5^{\circ}$ and $\sigma_{l}=15^{\circ}$ to take into account the bulge dimensions that followed from the bulge IR emission analysis (Dwek et al. 1995). In total, the map contains 4 excesses in the region of the galactic bulge that we call - BP1 $\left(l=18^{\circ}\right.$, $\left.b=5^{\circ}\right), \operatorname{BP} 2\left(l=357^{\circ}, b=-7^{\circ}\right), \operatorname{BP} 3\left(l=355^{\circ}, b=10^{\circ}\right)$ and Aquila $\left(l=28.5^{\circ}, b=-11.5^{\circ}\right)$. After smoothing, the BP1-BP3 excesses appear concentrated towards the galactic bulge. The spectra of BP1 and BP2 (shown in Figs. 10 and 11 of Sect. 3) clearly show a line at $\sim 1.27 \mathrm{MeV}$ (Table 1 ), as its strongest component, while the spectrum of BP3 has its strongest line at $\sim 1.36 \mathrm{MeV}$. It is conceivable that the BP3 excess may not be related to the galactic bulge at all, and could be a result of the projection effect of the $\gamma$-ray emission from the $\rho$ Oph region (note also the low significance of this excess: $2.2 \sigma$ only).

Aquila excess was detected as a point-like feature in our allsky map (Iyudin et al. 1999) with the likelihood value of $\sim 25.0$, that corresponds to $\sim 4.2 \sigma$ for 3 degrees of freedom (d.o.f.)

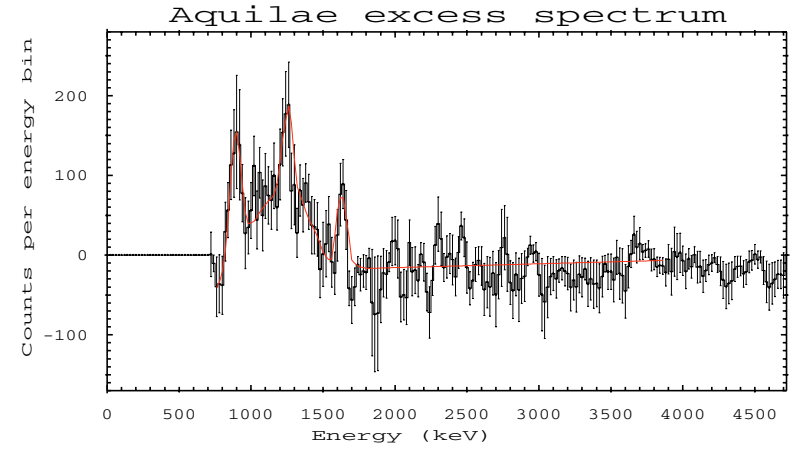

Fig. 3. The smoothed energy spectrum of the excess in the South Aquila region from $0.72 \mathrm{MeV}$ to $4.72 \mathrm{MeV}$ for the combination of observations from 2.0 to 624.1 clearly shows the $1.275 \mathrm{MeV}$ line. Two more lines at $0.89 \mathrm{MeV}$ and $\sim 1.6 \mathrm{MeV}$ are also visible in the fit shown by the solid line. All three lines are likely related to the both ${ }^{20} \mathrm{Ne}$ and ${ }^{22} \mathrm{Ne}$ isotopes abundance excess in this region. The line at $890 \mathrm{keV}$ has a $F W H M$ of $100 \mathrm{keV}$, and the significance of $3.9 \sigma$. Parameters of lines with energies $E_{\gamma}>1 \mathrm{MeV}$ are presented in Table 1 .

Table 1. Aquila and BP1, BP2, BP3 line energies for $E_{\gamma}>1 \mathrm{MeV}$, and their FWHM.

\begin{tabular}{r|ccc|ccc}
\hline \hline \multirow{2}{*}{ Excess } & $\begin{array}{c}E_{1} \\
\mathrm{keV}\end{array}$ & $\begin{array}{c}F W H M_{1} \\
\mathrm{keV}\end{array}$ & $\begin{array}{c}\text { Sign. } \\
\sigma\end{array}$ & $\begin{array}{c}E_{2} \\
\mathrm{keV}\end{array}$ & $\begin{array}{c}F W H M_{2} \\
\mathrm{keV}\end{array}$ & $\begin{array}{c}\text { Sign. } \\
\sigma\end{array}$ \\
\hline Aquila & 1260 & 110 & 3.4 & 1630 & 110 & 3.1 \\
BP1 & 1255 & 110 & 4.14 & 1780 & 60 & 2.2 \\
BP2 & 1250 & 120 & 3.4 & 1600 & 200 & 3.6 \\
BP3 & 1360 & 120 & 2.7 & & & \\
\hline
\end{tabular}

search, and is unlikely to be related to the bulge emission. The spectrum of the Aquila excess contains a line at $1.275 \mathrm{MeV}$, as well as two other lines at $\sim 0.9 \mathrm{MeV}$ and $1.63 \mathrm{MeV}$, that can be identified with the excitation lines of ${ }^{22} \mathrm{Ne}(0.89 \mathrm{MeV})$ and ${ }^{20} \mathrm{Ne}(1.634 \mathrm{MeV})$. From the ML map we have derived the following significances for BP1, BP2 and BP3 excesses if they are considered as the result of a point-like excess search with a 3 d.o.f. Namely, BP1 has a likelihood value of $16.4, \approx 3.2 \sigma$; $\mathrm{BP} 2$ has a likelihood value of 21.4 , which $\approx 3.9 \sigma$; and, finally, BP3 has a likelihood value of $9.8, \sim 2.2 \sigma$. Clearly, none of the $\mathrm{BP} 1, \mathrm{BP} 2$, and BP3 excesses in the 1.275 MeV COMPTEL ML map can be claimed as individually significant detection of a point-like excess in the all-sky map.

\subsection{The Aquila excess}

In terms of the likelihood ratio the Aquila excess is the most significant in the all-sky $1.275 \mathrm{MeV}$ line emission map. It is detected from the south Aquila region with a likelihood ratio of $\sim 25$ (Fig. 2). The spectrum of the Aquila excess contains the $1.275 \mathrm{MeV}$ line, as well as two other lines at $\sim 0.9 \mathrm{MeV}$ and $1.63 \mathrm{MeV}$ that can be identified with the excitation lines of ${ }^{22} \mathrm{Ne}$ and of ${ }^{20} \mathrm{Ne}$ (see Fig. 3).

In order to verify our spectral analysis approach and the background model we have also produced a spectrum for the region with coordinates $l=70^{\circ}, b=0^{\circ}$, that has no $1.275 \mathrm{MeV}$ line emission. One can clearly see the difference between the two spectra of Figs. 3 and 4, especially given that they are 


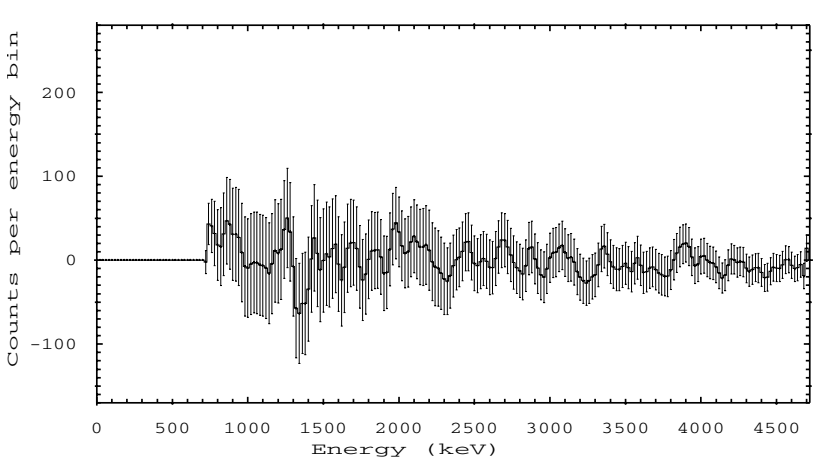

Fig. 4. The smoothed energy spectrum of the Cygnus region from $0.72 \mathrm{MeV}$ to $4.72 \mathrm{MeV}$ for the combination of observations from 2.0 to 601.1 , that clearly shows no line emission, if compared with the spectrum of Fig. 3. The same type of background was used as in Fig. 3 spectrum.

produced in the same way, and with the same background model. The spectrum of Fig. 4 has a rather strong variation around a line of the zero value of residual counts, but the maximum amplitude of such fluctuations does not exceed $30 \%$ of the $1.275 \mathrm{MeV}$ line amplitude of Fig. 3. This comparison supports our spectral analysis.

The flux of $\sim 3 \times 10^{-5} \mathrm{~cm}^{-2} \mathrm{~s}^{-1}$ in the $1.275 \mathrm{MeV}$ line measured from South Aquila (Figs. 1b and 2) can be produced in boundaries of different scenarios, among others with the scenario that relates a locally enhanced low-energy cosmic-ray intensity to the interaction region of the Local Bubble and Loop I (Egger \& Aschenbach 1995).

This interpretation is consistent with the persistency of the 1.275 MeV line emission from this region. No significant variation of the line flux value was detected during $\sim 6$ years exposure of this excess, which excludes its possible association with the exponentially decaying ${ }^{22} \mathrm{Na}$. The absence of the expected strong line at $4.4 \mathrm{MeV}$ from the Aquila excess is explained by the difficulty in handling the large instrumental background around this line (see Iyudin et al. 2004).

\subsection{The global distribution of $1.275 \mathrm{MeV}$ line emission and the excesses $\mathrm{BP} 1, \mathrm{BP} 2$ and $\mathrm{BP} 3$}

The global galactic distribution of the $1.275 \mathrm{MeV}$ line emission is based on the longitude and/or latitude profiles of the $1.275 \mathrm{MeV}$ line emissivity extracted from the maximumentropy (ME) map (Figs. 1a,b). The galactic distribution of $1.275 \mathrm{MeV}$ line emission was extracted from the ME-map assuming the shape of the galactic bulge to be a region of dimensions $|l| \leq 20^{\circ}$, and $|b| \leq 10^{\circ}$, that follows from the analysis of the COBE all-sky maps in the different IR wavelength bands (Weiland et al. 1994; Dwek et al. 1995; Freudenreich 1998). Interestingly, this selection of the bulge size leads to a maximum ratio of the extracted bulge flux relative to the flux of the halo region of the all-sky map in the $1.275 \mathrm{MeV} \gamma$-ray line, i.e. of the region that excludes the galactic plane with $|b| \leq 10^{\circ}$ (Fig. 5).

Distributions of the COMPTEL galactic 1.275 MeV line emission indicate a considerable bulge component of the

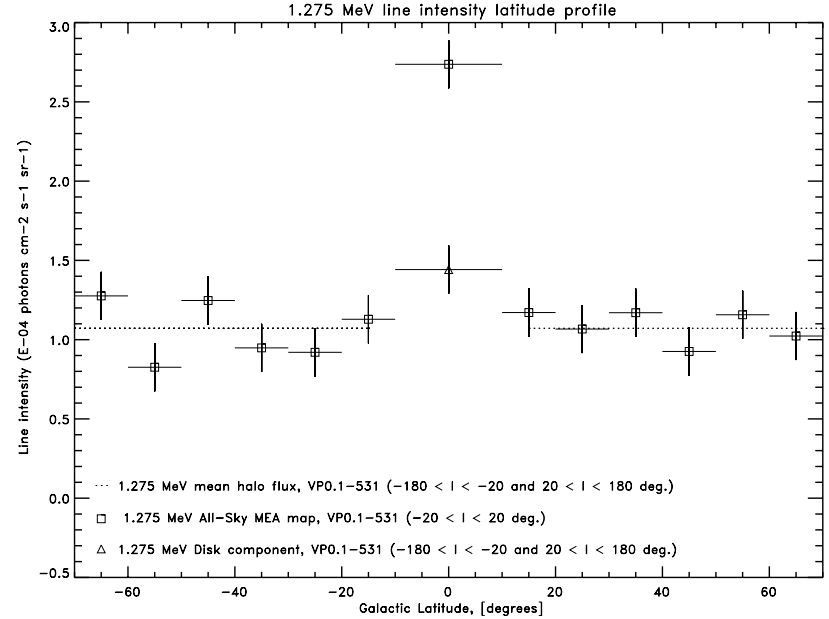

Fig. 5. The latitude profile of the galactic bulge region in the light of the $1.275 \mathrm{MeV}$ emission is shown by squares for the combination of all observations. The dotted line shows the mean halo intensity in the 1.275 MeV line as measured by COMPTEL. The triangle shows the disk component of the $1.275 \mathrm{MeV}$ line emission.

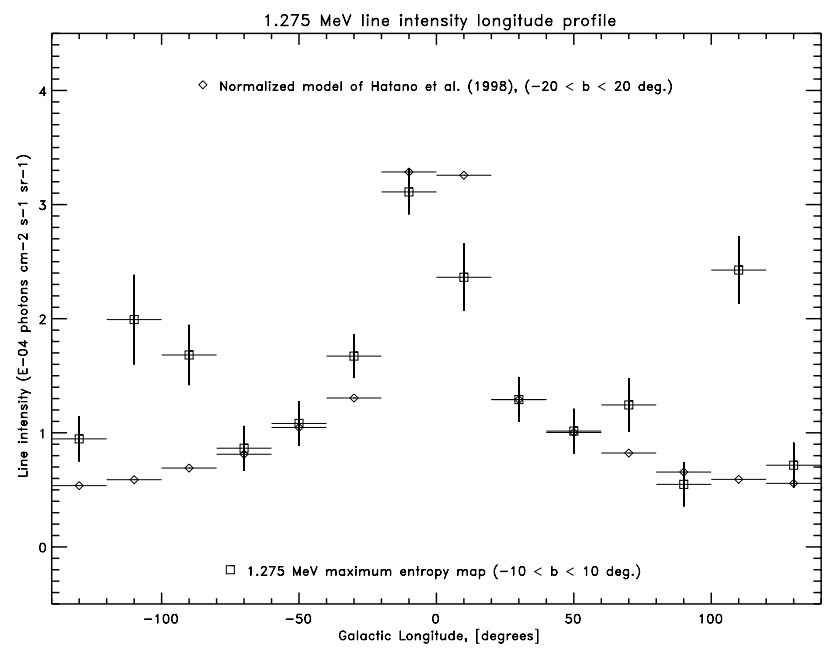

Fig. 6. The longitude profile of the galactic bulge region in the light of the $1.275 \mathrm{MeV}$ emission is shown by squares for the combination of all observations. Diamonds illustrate the shape of the novae longitude distribution as proposed by Hatano et al. (1997) which is normalised in the range of $|l| \leq 80^{\circ}$ to the data distribution.

Galactic 1.275 MeV line emission (Figs. 5-7). This component also dominates the ME-map of the galactic centre region in the $1.275 \mathrm{MeV}$ line emission (Fig. 8). The latitude profile of Fig. 5 shows an excess of the bulge $1.275 \mathrm{MeV}$ emission integrated over the bulge region with $|l| \leq 20^{\circ}$, and $|b| \leq 10^{\circ}$. The bulge component, shown by the square, clearly protrudes in the latitude distribution (Fig. 5), as well as in the longitude distribution (Fig. 6) of the $1.275 \mathrm{MeV}$ line flux. The error bars shown in Figs. 5-7 were derived from the scatter plot of the galactic halo $1.275 \mathrm{MeV}$ line flux distribution and, additionally, were verified by Monte-Carlo simulations of the all-sky 1.275 MeV line background models.

Figure 6 shows the $1.275 \mathrm{MeV}$ line longitude distribution, in which there is an overall drop of the $1.275 \mathrm{MeV}$ flux from the galactic centre towards values of $|l|$ up to $\sim\left(80^{\circ}\right.$ to $\left.100^{\circ}\right)$ where 


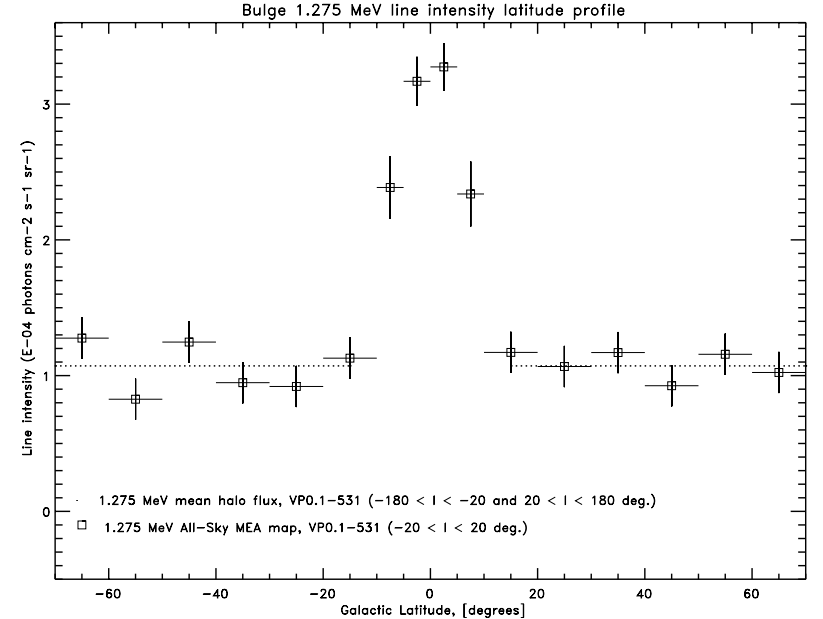

Fig. 7. The latitude profile of the galactic bulge region in the light of the $1.275 \mathrm{MeV}$ emission is shown by squares for the combination of all observations. Dotted line shows the mean halo intensity in the 1.275 MeV line as measured by COMPTEL.

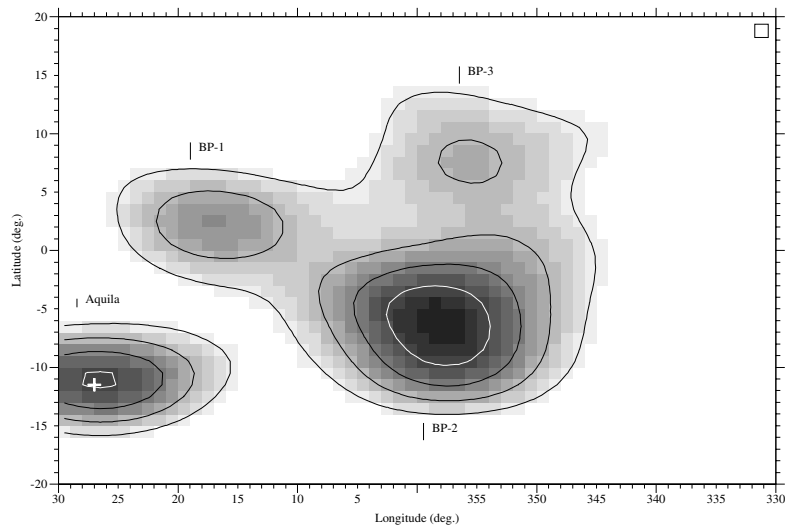

Fig. 8. The ME-map of the galactic centre region in the $1.275 \mathrm{MeV}$ line emission. COMPTEL observations up to April 1997 were used for this map. The position of Aquila excess is marked by the cross. Contour lines start at the intensity value of $1.7 \times 10^{-4}$ photons $\mathrm{cm}^{-2} \mathrm{~s}^{-1} \mathrm{sr}^{-1}$ with the step of $4.0 \times 10^{-5}$ photons $\mathrm{cm}^{-2} \mathrm{~s}^{-1} \mathrm{sr}^{-1}$.

it is interrupted by the excess emission at $l \sim 100^{\circ}$ to $120^{\circ}$, and $l \sim-80^{\circ}$ to $-120^{\circ}$. The largest excess at the longitude $\sim 110^{\circ}$ in the longitude distribution is partly explained by the contribution of the Cas A SNR flux in the line of ${ }^{44} \mathrm{Ti}$ at $1.157 \mathrm{MeV}$ (Iyudin 1999). Both energy intervals of the $1.157 \mathrm{MeV}$ line and of the $1.275 \mathrm{MeV}$ line that were used in COMPTEL studies are overlapping by $\sim 80 \mathrm{keV}$. Such an overlap is sufficient to produce an excess of $\sim 1.2 \times 10^{-4}$ photons $\mathrm{cm}^{-2} \mathrm{~s}^{-1} \mathrm{sr}^{-1}$ at the bin of $l \sim 110^{\circ}$ in the longitude distribution of the $1.275 \mathrm{MeV}$ line. Similarly the negative longitudes excess at $l \sim-80^{\circ}$ to $-120^{\circ}$ may be related to the G266.2-1.2 SNR flux (Iyudin et al. 1998) in the ${ }^{44} \mathrm{Ti} 1.157 \mathrm{MeV}$ line, with the rest of the excess being likely due to the Vela SNR. If one ignores, for the reasons given, these excesses at large positive and negative longitudes, then the overall shape of the distribution is quite consistent with the proposed model of Hatano et al. (1997). Note also, that the sum contribution of longitude distribution excesses at $|l|>80^{\circ}$
(Fig. 6), provides an explanation for the small excess of the "disk" component, shown by the triangle in Fig. 5.

The overall shape of the latitude and longitude distributions of the 1.275 line emission (Figs. 5 and 6) differs from the COMPTEL distributions for the continuum emission in the overlapping energy interval of $1.0-3.0 \mathrm{MeV}$ (Strong et al. 1997) and the ${ }^{26} \mathrm{Al}$ line flux distribution (Oberlack 1997). To illustrate this difference a distribution was produced with a fine binning in latitude (Fig. 7). The 1.0-3.0 MeV continuum emission latitude distribution has a full-width half-maximum value $(F W H M)$ of $\sim 5^{\circ}$, and the longitude distribution has a $F W H M_{\text {long }}^{1-3}$ of $\sim 90^{\circ}$, while the $1.275 \mathrm{MeV}$ line flux latitude distribution has $F W H M_{\text {lat }}^{1.275}$ of $\sim 16^{\circ}$ (Fig. 7), and the longitude distribution $F W H M_{\text {long }}^{1.275}$ is $\sim 45^{\circ}$ (Fig. 6). The same measures for the $1.8 \mathrm{MeV}$ galactic ${ }^{26} \mathrm{Al}$ related line emission have values of $F W H M_{\text {long }}^{1.8} \sim 75^{\circ}$, and $F W H M_{\text {lat }}^{1.8}=(3.9 \pm 1.2)^{\circ}$ (Oberlack 1997). The $1.275 \mathrm{MeV} \gamma$-ray line distribution is notably different from the both the galactic gamma-ray continuum or ${ }^{26} \mathrm{Al}$ line flux distributions, and is broadly consistent with the bulge shape derived from the COBE IR maps (Freudenreich 1998, and references therein).

The total flux of the $1.275 \mathrm{MeV}$ line bulge $\left(|b| \leq 10^{\circ} ;|l| \leq\right.$ $20^{\circ}$ ) component is

$I_{1.275}^{\text {bulge }}=\left[1.68 \pm(0.17)_{\text {stat }}\right] \times 10^{-4} \mathrm{ph} \mathrm{cm}^{-2} \mathrm{~s}^{-1} \mathrm{sr}^{-1}$.

This translates into a flux of:

$$
\begin{aligned}
F_{1.275}^{\text {bulge }}= & {\left[3.68 \pm(0.46) \pm(0.67)_{\text {syst }}\right] \times 10^{-5} \mathrm{ph} \mathrm{cm}^{-2} \mathrm{~s}^{-1}, } \\
& \text { again for }|b| \leq 10^{\circ} ;|l| \leq 20^{\circ} .
\end{aligned}
$$

This flux value is $\sim 30 \%$ smaller than the previously published upper limit of $8 \times 10^{-5} \mathrm{ph} \mathrm{cm}^{-2} \mathrm{~s}^{-1} \mathrm{rad}^{-1}$ by SMM (Leising et al. 1988).

\section{3. ${ }^{22} \mathrm{Ne}$ excitation by low-energy cosmic rays in the bulge}

In the following we assume that a large fraction of $\sim 1.27 \mathrm{MeV}$ line emission detected from the bulge is a product of the lowenergy cosmic ray interactions with the abundant elements of the galactic bulge ISM. The plausibility of such a scenario is dependent on the uncertain shape and composition of the lowenergy cosmic ray spectrum in the galactic bulge (Meneguzzi \& Reeves 1975; Yoshimori 1979), and on the amount of ${ }^{22} \mathrm{Ne}$ and its distribution in the galactic bulge.

The ${ }^{22} \mathrm{Ne}$ isotope is known to be copiously produced by the $\alpha$ capture on ${ }^{14} \mathrm{~N}$ in the low- (Gallino et al. 1998), and intermediate-mass AGB stars (Marigo et al. 1996; Forestini \& Charbonnel 1997), as well as by massive stars with $M \geq 25 M_{\odot}$ during their WC stage of the Wolf-Rayet (WR) evolutionary sequence (Maeder \& Meynet 1993; Meynet et al. 2001). It is noteworthy that the density distribution of AGB and WR stars increases towards the galactic centre (Weinberg 1992; Conti \& Vacca 1990).

We recall here, that COMPTEL's $1.275 \mathrm{MeV}$ line-intensity profiles (Figs. 6 and 7) are consistent with the bulge shape having the major-to-minor bulge axis ratio of $\sim 2$. This ratio is consistent with the bulge model derived from the COBE 


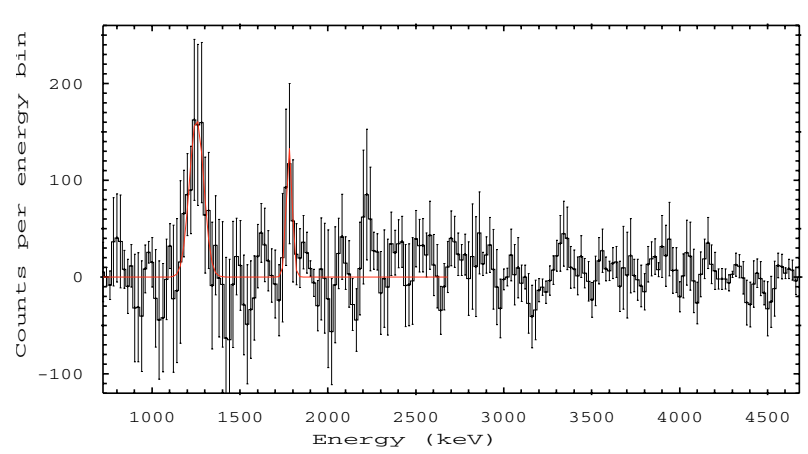

Fig. 9. The smoothed spectrum of the galactic bulge P1 region clearly shows the $\sim 1.27 \mathrm{MeV}$ line. All COMPTEL's observations up to April 1997 were used in producing this spectrum. Also seen is the trace of the galactic $\sim 1.8 \mathrm{MeV}$ line, that is suppressed due to the method used for the spectrum derivation.

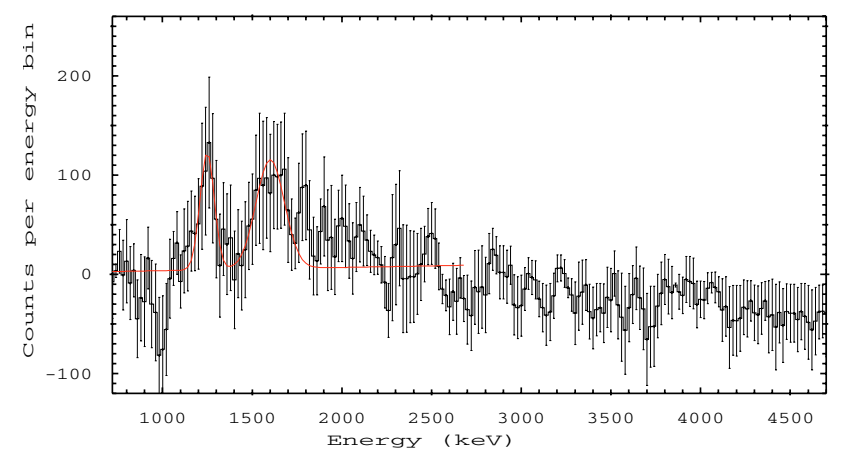

Fig. 10. The smoothed spectrum of the galactic bulge $\mathrm{P} 2$ region clearly shows the $\sim 1.27 \mathrm{MeV}$ line emission. Also seen is the blend of the excitation lines at $\sim 1.6 \mathrm{MeV}$, and at $\sim 1.8 \mathrm{MeV}$, fitted as a single broad feature.

measurements of the galactic IR-emissivity (Dwek et al. 1995; Freudenreich 1998), but contradicts the usual assumption of a spherical galactic bulge (Higdon \& Fowler 1987; Hatano et al. 1997).

These facts support the $1.275 \mathrm{MeV}$ line production scenario in the galactic bulge by the excitation of ${ }^{22} \mathrm{Ne}$, however we cannot exclude possible foreground contributors to the projected intensity map. In fact, the spectra of prominent excesses in the 1.275 MeV map have rather different appearances (Figs. 9 and 10), the differences possibly being related to the contribution of foreground objects.

Based on the He abundance measurements in the galactic bulge, which is established at the level of $Y=0.28 \pm$ 0.02 (Minniti 1995), we selected the ratio of $K_{\alpha} / K_{\mathrm{H}}$ (from Meneguzzi \& Reeves 1975) equal to the mean galactic cosmic rays value of $4 \%$. This ratio leads to an increase of the expected $\gamma$-ray line flux from the GC region by a factor of 3.2 for the steep low-energy cosmic ray spectrum with $\gamma=5$, compared to the estimate of Meneguzzi \& Reeves (1975). By using 1.275 MeV line production cross sections of reactions ${ }^{22} \mathrm{Ne}(\mathrm{p}, \mathrm{n}){ }^{22} \mathrm{Na}$ (Saam et al. 1989) and ${ }^{22} \mathrm{Ne}(\mathrm{p}$, $\left.\mathrm{p}^{\prime} \gamma_{1.275}\right)^{22} \mathrm{Ne}^{*}$ (Sorokin et al. 1963) we evaluated the expected $\gamma$-ray line flux from the galactic bulge following the approach of Meneguzzi \& Reeves (1975). For a cosmic ray spectrum with the shape $\sim E^{-5}$ the source function $q(E)$ for the line production by cosmic ray protons reaches a value of $\sim 4 \times$ $10^{-27}$ photons ( $\mathrm{H}$ atom) $)^{-1} \mathrm{~s}^{-1} \mathrm{MeV}^{-1} \mathrm{sr}^{-1}$. By taking into account the $\alpha$ particles input into the line production, we finally get a flux from the galactic centre region of the order of

$F_{\gamma}(E) \sim 1.0 \times 10^{-3} \mathrm{~cm}^{-2} \mathrm{~s}^{-1} \mathrm{sr}^{-1} \mathrm{MeV}^{-1}$.

This flux estimate is uncertain by as much as one order of magnitude, but it is close to the value of $\sim 1.7 \times 10^{-4}$ photons $\mathrm{cm}^{-2} \mathrm{~s}^{-1} \mathrm{sr}^{-1}$, that we have derived for the $1.275 \mathrm{MeV}$ line $(F W H M \sim 110 \mathrm{keV})$ flux from the galactic bulge. The uncertainties of our estimate are due to the uncertain composition of the low-energy cosmic rays, as well any other contribution to the $1.275 \mathrm{MeV}$ line production on targets different from the ${ }^{22} \mathrm{Ne}$, which have been neglected.

The above results are by no means final, but they do point to the interesting possibility of deriving the shape of the galactic bulge, and the low-energy cosmic ray density distribution in the Galaxy, by more sensitive measurements of the global galactic distributions of $1.275 \mathrm{MeV}$ and other excitation $\gamma$-ray lines with future Compton-type telescopes like MEGA and ACT.

\section{Conclusions}

The possible detection of 1.275 MeV $\gamma$-ray line emission from the Galactic bulge region by COMPTEL questions the origin of the major contributor to the $1.275 \mathrm{MeV}$ line emission from the bulge. Whether the extended $1.275 \mathrm{MeV}$ line emission origin can be explained by the ${ }^{22} \mathrm{Na}$ accumulation in the bulge, or by the cosmic ray excitation of the ${ }^{22} \mathrm{Ne}$, remains an open question. The latter alternative is our preferable interpretation, i.e. a scenario that explains the bulge excess of the $1.275 \mathrm{MeV}$ line emission by low-energy cosmic ray interactions with the abundant ${ }^{22} \mathrm{Ne}$ in the bulge. If this is the case, then a major part of the hard X-ray emission in the galactic ridge detected by ROSAT (Tanaka et al. 1999), ASCA (Sugizaki et al. 2001), and Chandra (Ebisawa et al. 2001) can be explained by the cosmic ray interactions with the gaseous matter of the bulge.

We note though, that above conclusion should be taken cautiously in view of the systematic uncertainties of COMPTEL 1.275 MeV line flux estimate from the galactic bulge.

Acknowledgements. We acknowledge then comments of the anonymous referee that have served to make the paper more concise. The COMPTEL project was supported by the German "Ministerium für Bildung und Forschung" through DLR grant 50 QV 9096 8. A.F.I. acknowledges financial support from the German "Ministerium für Bildung und Forschung" through the DLR grant 50 OR 0002.

\section{References}

D’Auria, J.M., Azuma, R. E., Bishop, S., et al. 2004, Phys. Rev., C69, 065803

Bishop, S., Azuma, R. E., Buchmann, L., et al. 2003, Phys. Rev. Lett., 90, 162501

Bloemen, H., Hermsen, W, Swanenburg, B. N., et al. 1994, ApJS, 92, 419

Bloemen, H. et al. 1999, in Proc. of 5th Compton Symposium

Clayton, D. D., \& Hoyle, F. 1974, ApJ, 187, L101

Conti, P. S., \& Vacca, W. D. 1990, AJ, 100, 431 
Dwek, E., Arendt, R. G., Hauser, M. G., et al. 1995, ApJ, 445, 716

Ebisawa, K., Maeda, Y., Kaneda, H., \& Yamauchi, S. 2001, Science, 293, 1633

Egger, R., \& Aschenbach, B. 1995, A\&A, 294, L25

Forestini, M., \& Charbonnel, C. 1997, A\&AS, 123, 241

Freudenreich, H. T. 1998, ApJ, 492, 495

Gallagher, J. S., \& Starrfield, S. 1978, ARA\&A, 16, 171

Gallino, R., Arlandini, C., Busso, M., et al. 1998, ApJ, 497, 388

Gehrz, R. D., Truran, J. W., Williams, R. E., \& Starrfield, S. 1998, PASP, 100, 3

Gomez-Gomar, J., Hernanz, M., Jose, J., \& Isern, J. 1998, MNRAS, 296, 913

Harris, M. J., Teegarden, B. J., Cline, T. L., et al. 2000, ApJ, 542, 1057

Hatano, K., Branch, D., Fisher, A., \& Starrfield, S. 1997, MNRAS, 290,113

Hernanz, M., Gomez-Gomar, J., Jose, J., Coc, A., \& Isern, J. 1999, Astrophys. Lett. \& Comm., 38, 407

Higdon, J. C., \& Fowler, W. A. 1987, ApJ, 317, 710

Iliadis, C., Champagne, A., José J., Starrfiels, S., \& Tupper, P. 2002, ApJS, 142, 105

Iyudin, A. F., Diehl, R., Bloemen, H., et al. 1994, A\&A, 284, L1

Iyudin, A. F., Bennett, K., Bloemen, H., et al. 1995, A\&A, 300, 422

Iyudin, A. F., Schönfelder, V., Bennett, K., et al. 1998, Nature, 396, 142

Iyudin, A. F., Bennett, K., Bloemen, H., et al. 1999, Astrophys. Lett. \& Com., 38, 371

Iyudin, A. F., Bennett, K., Bloemen, H., et al. 2000, AIP, 510, 92

Iyudin, A. F., Böhringer, H., Dogiel, V. A., \& Morfill, G. 2004, A\&A, 413,817

Jenkins, D. G., Lister, C. J., Janssens, R.V., et al. 2004, Phys. Rev. Lett., 92, 031101

José, J., \& Hernanz, M. 1998, ApJ, 494, 680
Jose, J., Coc, A., \& Hernanz, M. 1999, ApJ, 520, 347

Leising, M. D., Share, G. H., Chupp, E. L., \& Kanbach, G. 1988, ApJ, 328,755

Leising, M. D., et al. 1993, AIP CP-280, 137

Leising, M. D. 1997, AIP, CP-410, 163

Maeder, A., \& Meynet, G. 1993, A\&A, 278, 406

Mahoney, W. A., Ling, J. C., Jacobson, A. S., \& Lingenfelter, R. E. 1982, ApJ, 262, 742

Marigo, P., Bressan, A., \& Chiosi, C. 1996, A\&A, 313, 345

Meneguzzi, M., \& Reeves, H. 1975, A\&A, 40, 91

Meynet, G., Arnould, M., Paulus, G., \& Maeder, A. 2001, SSRv, 99, 73

Minniti, D. 1995, A\&A, 300, 109

Oberlack, U. 1997, Ph.D. Thesis, TU Munich, Germany

Saam, B., Skalsey, M., \& van House, J. 1989, Phys. Rev., C40, 1563

Schönfelder V., Aarts H., Bennet K., et al. 1993, ApJS, 86, 657

Sorokin, P. V., et al. 1963, Sov. JETP, 16, 529

Starrfield, S., Sparks, W. M., Truran, J. W., \& Wiescher, M. C. 2000, ApJS, 127, 485

Strong, A. W., Diehl, R., Schonfelder, V., et al. 1997, AIP CP-410, 1198

Sugizaki, M., Matsuda, K., Kaneda, H., et al., 2001, ApJS, 134, 77

Tanaka, Y., Miyaji, T., \& Hasinger, G. 1999, Astron. Nachr., 320, 181

Truran, J. W. 1982, in Essays in Nuclear Astrophysics, ed. C. A. Barnes, D. D. Clayton, \& D. N. Schramm (Cambridge: Cambridge Univ. Press), 467

Weiland, J. L., Arendt, R. G., Berriman, G. B., et al. 1994, ApJ, 425, 81

Weinberg, M. D. 1992, ApJ, 384, 81

Woosley, S. E., \& Weaver, T. A. 1995, ApJS, 101, 181

Yoshimori, M. 1979, Aust. J. Phys., 32, 383 